\title{
Colorimetric measurement of iron in plasma samples anticoagulated with EDTA
}

\author{
T A Walmsley, P M George, R T Fowler
}

\begin{abstract}
Aims: To determine if the iron in EDTA anticoagulated plasma samples can be measured by colorimetric assays using Ferrozine.

Methods: Paired samples of serum and EDTA plasma were obtained from 24 patients and analysed by three commercial iron methods. The EDTA plasmas were also analysed using methods modified by the addition of zinc sulphate or with different concentrations of Ferrozine. The iron contamination of EDTA sample tubes was measured by atomic absorption spectroscopy.

Results: Two commercial colorimetric iron methods gave results of zero for EDTA plasma samples. A third commercial reagent gave plasma results that were about $30 \%$ lower than their corresponding serum samples. Addition of $7 \mathrm{mmol} / 1$ zinc sulphate to this reagent system and extending the sample preincubation time to 300 seconds yielded comparable results from paired serum and EDTA plasma samples. Linear regression analysis gave a slope of 0.97 with an intercept of $0.60 \mu \mathrm{mol} / 1$ and $R^{2}=0.9943$. Measurements by atomic absorption spectroscopy showed that this positive intercept was due to contamination of the blood collection tubes with about $90 \mathrm{ng}$ of iron.

Conclusions: Modification of commercial colorimetric iron methods permits the biochemical assessment of iron status and a full blood count from a single EDTA anticogulated blood sample.
\end{abstract}

In clinical practice a patient's iron stores are often assessed by the analysis of serum iron, transferrin, and ferritin concentrations in conjunction with a full blood count measured on a blood sample anticoagulated with EDTA. Previous work has shown that the collection of the anticoagulated contents of the EDTA tube before that of the whole blood tube can leave traces of EDTA on the subsequent tube. ${ }^{2}$ Even at this low level of EDTA contamination in the serum sample, the iron is chelated by the EDTA and its reaction with the Ferrozine colorimetric reagent is inhibited, producing erroneously low serum iron concentrations. This type of EDTA interference can be prevented by the addition of zinc sulphate to the ascorbic acid reagent. ${ }^{2}$ During sample preincubation with the ascorbic acid reagent, the zinc ions preferentially bind to the EDTA and leave the ferrous ions free to react with the Ferrozine reagent. In this situation the concentration of EDTA in the contaminated serum samples is unlikely to exceed $0.2 \mathrm{mmol} / 1$ and a zinc sulphate concentration of $0.5 \mathrm{mmol} / \mathrm{l} \mathrm{effec}$ tively prevents the interference resulting from EDTA chelating the serum iron.

Obviously, it would sometimes be desirable to measure the plasma iron concentration in the same sample that is used for the full blood count. For example, whenever the full blood count unexpectedly suggests iron deficiency the plasma could be separated from the full blood count sample and analysed for iron, saving both time and money: there would be no need to recall the patient for a further blood sample. The routine use of a single sample for a full blood count and plasma iron would also minimise the blood volume required for these analyses and halve the number of sample collection tubes.

We describe a modification of a colorimetric iron method that permits the accurate measurement of iron in plasma samples anticoagulated with EDTA.

\section{Methods}

The iron concentration was measured in EDTA plasma from 24 patients with a range of serum iron concentrations from 5 to $33 \mu \mathrm{mol} / 1$. Paired samples of serum and EDTA plasma were collected at the same draw from patients presenting for "iron studies" and the results compared by linear regression. ${ }^{3}$ Serum samples were prepared from whole blood samples collected into $10 \mathrm{ml}$ draw Becton Dickinson Vacutainers without anticoagulant (Becton Dickinson Vacutainer Systems, Rutherford, New Jersey, USA; product number 6430 , lot number 8J713). These blood samples were allowed to clot for 30 minutes before the serum was collected and centrifuged. EDTA anticoagulated blood samples were collected in Becton Dickinson Vacutainer EDTA tubes containing $50 \mu \mathrm{l}$ tripotassium EDTA $(15 \%)$ in a $75 \mathrm{~mm} \times 13 \mathrm{~mm}$ diameter tube with a draw volume of $5 \mathrm{ml}$ (product number 6452, lot number 9K067). To prevent EDTA carryover into the serum sample the EDTA anticoagulated blood sample was always collected after the whole blood sample. For the purpose of calculations it was assumed that $5 \mathrm{ml}$ of blood collected into these tubes contained $2.5 \mathrm{ml}$ of plasma and $7 \cdot 5 \mathrm{mg}$ EDTA (7.3mmol/1 EDTA). Venoject collection tubes were obtained from Terumo Medical corporation, Elkton, Maryland, USA. 
We measured iron in serum and EDTA anticoagulated plasma samples by five methods based on the Ferrozine colorimetric reaction. ${ }^{4}$ Method A: using a SMAC analyser (Technicon Instruments Corp., Tarrytown, New York, USA; method 5G4-0025 FH9).

Method B: using a Hitachi 717 analyser with Boehringer reagents (Boehringer Mannheim, West Germany; method 1040880).

Method C: using a Cobas Bio centrifugal analyser (Hoffman La Roche \& Co Ltd., Basle, Switzerland; method 0710598). In this method $50 \mu \mathrm{l}$ of plasma and $30 \mu \mathrm{l}$ of deionised water is preincubated for 60 seconds at $25^{\circ} \mathrm{C}$ with $125 \mu \mathrm{l}$ of guanidine hydrochloride $(4.5 \mathrm{~mol} / \mathrm{l}$, pH 5.0) containing ascorbic acid ( $5 \mathrm{~g} / \mathrm{l})$. After the initial absorbance at $562 \mathrm{~nm}$ is read, $10 \mu \mathrm{l}$ of Ferrozine reagent $(40 \mathrm{mmol} / \mathrm{l})$ is added and the final absorbance is read after a further incubation of 300 seconds.

This Roche method (Method C) was also modified by adding $2 \mathrm{~g} / 1(7 \cdot 7 \mathrm{mmol} / \mathrm{l})$ zinc sulphate heptahydrate (AR Grade, BDH Laboratory Chemicals, Poole, England) to the guanidine/ascorbic acid reagent and preincubating the sample with this modified reagent for either 60 seconds (Method D) or 300 seconds (Method E). The concentration of zinc sulphate added to the Roche reagent $(7 \cdot 0 \mathrm{mmol} / \mathrm{l})$ was calculated to equal twice the molar concentration of EDTA present in the reagent after the addition of a plasma sample containing EDTA.

To determine the level of iron contamination in the EDTA collection tubes we half-filled the tubes with deionised water and measured this solution by atomic absorption spectroscopy at $248.3 \mathrm{~nm}$ using a Varian AA10 (Varian Techtron Pty. Limited, Mulgrave, Victoria 3170, Australia) and an air acetylene flame. ${ }^{5}$ The iron concentration measured in the aqueous solution was assumed to be equivalent to the iron concentration contaminating the plasma sample separated from a full blood tube.

To investigate the effect of the different compositions of the Boehringer (Method B) and Roche (Methods $\mathrm{C}$ to $\mathrm{E}$ ) reagents (table 1), we analysed two samples for iron using reagents modified by the addition of thiourea (Roche) or zinc sulphate and with different Ferrozine concentrations.

\section{Results}

COMPARISON OF EDTA AND SERUM SAMPLES

The results obtained by analysing 24 paired serum samples and EDTA plasma samples for iron by four methods ( $A$ to $C$ and $E$ ) are summarised in fig 1 . Both the SMAC analyser (Method A) and the Boehringer Hitachi 717 (Method B) gave results of zero for all the

Table 1 Reagent concentrations in the final colorimetric iron reactions

\begin{tabular}{lcc}
\hline & Roche & Boehringer \\
\hline Hydrogen ions & $10 \mu \mathrm{mol} / 1$ & $10 \mu \mathrm{mol} / 1$ \\
Hydrochloride & $2.6 \mathrm{~mol} / 1$ & $3.5 \mathrm{~mol} / 1$ \\
Thiourea & $0 \mathrm{mmol} / 1$ & $78 \mathrm{mmol} / 1$ \\
Ferrozine & $1.9 \mathrm{mmol} / 1$ & $0.27 \mathrm{mmol} / 1$ \\
\hline
\end{tabular}

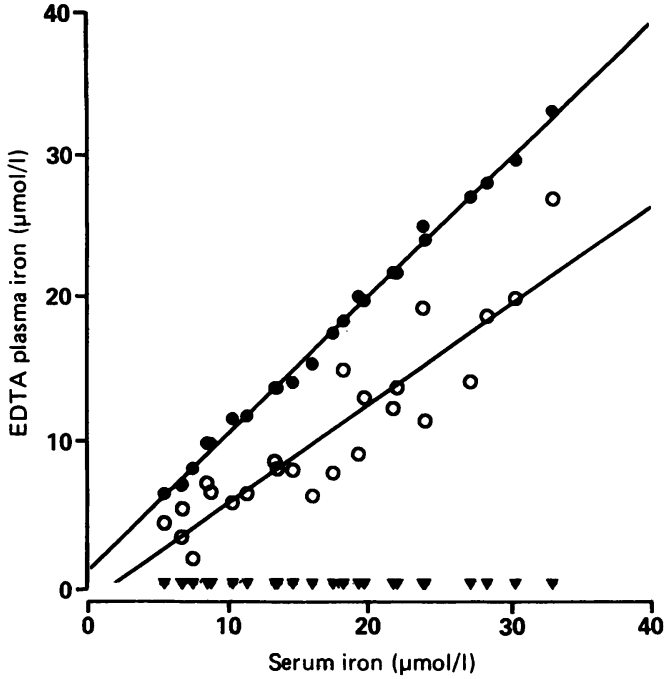

Figure 1 Comparison of serum iron and EDTA anticoagulated plasma iron. SMAC (Method $A$ ) and Hitachi 717 (Method B); $\bigcirc$ Unmodified Roche reagent (Method C); Modified Roche reagent (Method E)sample preincubated for 300 seconds with $7 \mathrm{mmol} / \mathrm{l}$ zinc sulphate added to guanidine/ascorbic acid reagent.

EDTA plasma samples. The results obtained with the unmodified Roche method (Method C) showed that the iron concentrations measured in the EDTA plasma samples were always lower than the corresponding serum samples (mean difference $-6 \cdot 2 \mu \mathrm{mol} / 1$ ). Analysis of these results by linear regression gave a slope of 0.69 and an intercept of $-1.0 \mu \mathrm{mol} / 1$ $\left(R^{2}=0 \cdot 8458\right)$. This slope was significantly less than $1.0(p<0.05)$. Addition of $7.0 \mathrm{mmol} / 1$ zinc sulphate to the guanidine/ascorbic acid reagent (Method D) increased the iron response from the EDTA plasma samples and gave a mean difference of $-1 \cdot 1 \mu \mathrm{mol} / 1$ plus an improved correlation $\left(R^{2}=0.9573\right)$ with a slope of 0.94 and an intercept of $0.0 \mu \mathrm{mol} / 1$. Increasing the preincubation time of the sample with the modified zinc/guanidine/ascorbic acid reagent to 300 seconds (Method $E$ ) further improved the correlation $\left(R^{2}=0.9943\right)$. A similar extension of the preincubation time but without the inclusion of zinc in the reagent gave results similar to those obtained with Method C (data not shown). With these two modifications (Method E), the mean difference between serum and EDTA samples was $+0.15 \mu \mathrm{mol} / 1$ with most of the EDTA plasma results being higher than the corresponding serum samples. Analysis of these results by linear regression gave a slope of 0.97 and an intercept of $0.60 \mu \mathrm{mol} / 1$. The intercept was significantly greater than zero $(p<0.05)$ indicating that the EDTA collection tubes may have been contaminated with iron

MEASUREMENT OF IRON CONTAMINATION IN EDTA COLLECTION TUBES

We measured the iron contamination in 10 EDTA tubes (Becton Dickinson Vacutainer; product number 6452 , lot number 9K067). The mean iron concentration was $0.63 \mu \mathrm{mol} / 1$ indicating that the plasma from $5 \mathrm{ml}$ of whole blood collected into these tubes will be contaminated with $0.63 \mu \mathrm{mol} / 1$ of iron. This is 
Table 2 Iron contamination in EDTA anticoagulated collection tubes

\begin{tabular}{lllcc}
\hline Manufacturer & Lot No & Anticoagulant & $\begin{array}{l}\text { Volume } \\
(\boldsymbol{m l})\end{array}$ & $\begin{array}{l}\text { Iron } \\
(\boldsymbol{\mu m o l} / \mathbf{l})\end{array}$ \\
\hline Vacutainer & 9J111 & EDTA K3 & 2 & 0.5 \\
Venoject & LL1323 & EDTA Na2 & 5 & 1.9 \\
Venoject & LP0124 & EDTA K3 & 5 & 1.8 \\
Venoject & MH0624 & EDTA K3 & 5 & 2.5 \\
Vacutainer & 9G102 & EDTA K3 & 7 & 1.5 \\
Vacutainer & 9K079 & EDTA K3 & 7 & $0 \cdot 8$ \\
Vacutainer & 9C120 & EDTA K3 & 7 & 0.6 \\
Venoject & LM2424 & EDTA K3 & 7 & 1.8 \\
Venoject & LG27141A4 & EDTA K3 & 10 & 2.0 \\
\hline
\end{tabular}

Figure 2 Effect of the volume of blood collected into EDTA anticoagulated blood tube on the plasma iron concentration. $O$ Measured plasma iron by Method E; $\bigcirc$ plasma iron corrected for $a$ contamination of $90 \mathrm{ng}$ of iron in the EDTA anticoagulated blood

tubes; - - - - iron concentration of corresponding serum sample.

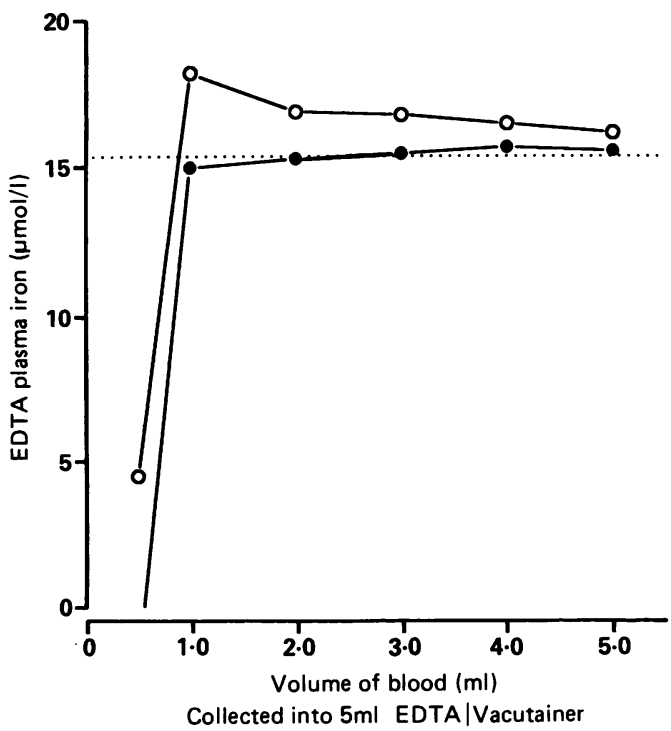

equivalent to the intercept observed with Method $\mathrm{E}$ and equates to an iron contamination of $90 \mathrm{ng}$ per tube. To establish the extent of iron contamination in other collection tubes we determined the iron contamination in nine different batches of blood collection tubes using atomic absorption spectroscopy. The range of iron contamination found was $0.5-2.5 \mu \mathrm{mol} / 1$ (table 2).

\section{EFFECT OF SAMPLE VOLUME ON IRON} CONCENTRATIONS IN EDTA PLASMA

To assess the effect of sample volume we measured the iron concentrations in plasma from underfilled $5 \mathrm{ml}$ EDTA blood collection tubes. Six plasma samples were prepared from tubes containing $5 \mathrm{ml}, 4 \mathrm{ml}, 3 \mathrm{ml}, 2 \mathrm{ml}, 1 \mathrm{ml}$ and $0.5 \mathrm{ml}$ of whole blood. In these samples the calculated plasma concentrations of EDTA $7 \cdot 3,9 \cdot 3,12 \cdot 2,18 \cdot 3$, and $73.3 \mathrm{mmol} / 1$, respectively. The apparent iron concentrations in these samples were measured using Method E.

Table 3 Effect of EDT A on iron analysis using modified commercial reagents

\begin{tabular}{lll}
\hline & \multicolumn{2}{c}{$\%$ Response from EDT A plasma compared with serum } \\
\cline { 2 - 3 } Reagent modification & Boehringer & Roche \\
\hline Standard conditions & 0 & 69 \\
+ Zinc $(7 \mathrm{mM})$ & 0 & 95 \\
+ Thiourea $(100 \mathrm{mM})$ & NA & 70 \\
+ Zinc and thiourea & NA & 97 \\
Ferrozine $(0.27 \mathrm{mM})$ & 0 & 0 \\
Ferrozine $(1.8 \mathrm{mM})$ & 67 & 69 \\
Ferrozine $(6.0 \mathrm{mM})$ & 97 & NT \\
\hline
\end{tabular}

NA Not applicable

NT Not tested
The results are summarised in fig 2 which shows that the measured plasma iron concentrations increased as the sample volume decreased from $5 \mathrm{ml}$ to $1 \mathrm{ml}$ and then sharply decreased at a sample volume of $0.5 \mathrm{ml}$. At a $0.5 \mathrm{ml}$ sample volume, the EDTA concentration is 10 times higher than the $5 \mathrm{ml}$ sample and the iron response is suppressed due to chelation. As the blood collection tubes are contaminated with $90 \mathrm{ng}$ of iron underfilling will result in an increased plasma iron concentration of $0.63,0.79,1.05,1.58,3.15$ and $6.30 \mu \mathrm{mol} / 1$, respectively. For blood volumes of $\geqslant 1 \mathrm{ml}$, correcting the measured plasma iron result for this contamination gives a plasma iron concentration that is comparable with the corresponding serum iron result (fig 2 ).

\section{EFFECT OF VARIATION IN THE REAGENT} COMPOSITION ON IRON CONCENTRATIONS MEASURED IN EDTA PLASMA

Table 1 shows that there are two major differences in the composition of the Roche and Boehringer reagents. The Boehringer reagent contains thiourea and a much lower concentration of Ferrozine. As the Boehringer reagent gave no response to iron in EDTA samples (fig 1 ), we tested the effect of adding zinc and Ferrozine to this reagent (table 3 ). In contrast to the Roche reagent, the addition of zinc did not abolish the EDTA effect, but increasing the Ferrozine concentration to that in the Roche reagent $(1.9 \mathrm{mM})$ resulted in a similar response from both methods, and a further increase in the Ferrozine concentration almost abolished the EDTA effect. The addition of thiourea to the Roche reagent had no clinically relevant impact.

\section{Discussion}

Because samples for iron studies are often collected in conjunction with an EDTA sample for a full blood count any interference as a result of iron chelation is undesirable. Previous work on the interference of EDTA in colorimetric iron assays documents the effect of EDTA contamination at a concentration of $0.2 \mathrm{mmol} / 1$ in serum samples collected after an EDTA sample. ${ }^{2}$ These studies showed that the addition of $0.5 \mathrm{mmol} / 1$ of zinc sulphate to the ascorbic acid reagent prevents this type of EDTA interference. In contrast, the EDTA in plasma collected for a full blood count is about 40-fold higher (7.3 mmol/1).

At $\mathrm{pH} \mathrm{7,} \mathrm{the} \mathrm{stability} \mathrm{constant} \mathrm{of} \mathrm{the} \mathrm{zinc}$ sulphate, EDTA binding is 100 -fold greater than for the iron EDTA complex. ${ }^{6}$ In contrast, Ferrozine preferentially binds to iron. Therefore the addition of excess zinc sulphate to an iron reagent will enhance the formation of the iron Ferrozine complex even in the presence of EDTA. We added zinc sulphate ( $7 \mathrm{mmol} / \mathrm{l}$ ) to a Roche reagent to equal twice the molar concentration of EDTA present in the reaction after the addition of a plasma sample containing $7.3 \mathrm{mmol} / 1 \mathrm{EDTA}$. In this phase of the reaction the release of iron from transferrin is accelerated by guanidine hydrochloride, and the added zinc ions competitively inhibit chela- 
tion of the released iron by EDTA. Therefore, when Ferrozine is added, the iron is free to react. Due to the lower concentrations of Ferrozine, however, the addition of zinc had no effect on the Boehringer reagent. Although an increase in the concentration of Ferrozine to $6.0 \mathrm{mM}$ abolishes the EDTA effect, it is both more convenient and much cheaper to prepare a modified reagent by the addition of zinc sulphate.

All the evacuated EDTA anticoagulated collection tubes that we tested contained iron contamination. Thus laboratories that wish to measure iron in EDTA plasma should be mindful of this potential problem and measure the level of contamination in their own batches of EDTA tubes. With the knowledge that plasma iron can now be measured in plasma from these tubes the manufacturers may be able to produce tubes free of iron contamination. However, where the observed contamination level is low, this can be ignored as it results in a minimal systematic error when the collection tubes are filled. When the tubes are underfilled the plasma iron concentration apparently increases until at a blood volume of $<1 \mathrm{ml}$ per $5 \mathrm{ml}$ tube even the modified reagent is unable to prevent the interference due to the very high concentration of EDTA present. Fortunately, this type of underfilled sample is also unsuitable for the full blood count and in practice should not be commonly encountered.
Our modified reagent system effectively overcomes the EDTA interference and permits the accurate measurement of plasma iron in samples being used for a full blood count. To perform complete iron studies on this sample the iron saturation can be calculated using the transferrin concentration measured by an immunoassay. While ferritin can also be measured in EDTA plasma, it must be remembered that EDTA interferes with its measurement by several enzyme immunoassays that utilise alkaline phosphatase as the label. By using the appropriate immunoassays in combination with a commercial iron reagent modified by the inclusions of zinc, however, it is possible to use a single sample for a full blood count plus full iron studies. This has practical advantages and should result in savings of both time and money.

1 Iron deficiency and iron overload. In: Callendar ST, ed. Clinics in haematology Volume 2. London: WB Saunders Co Ltd, 1973.

2 Koopman BJ, Hindriks FR, Lokerse YG, Wothers BG Orverdijk JK. Injurious effect of EDTA contamination on colorimetry of serum iron. Clin Chem 1985;31:2030-2.

3 Westgard JO, de Vos DJ, Hunt MR, Quam EF, Garber CC Carey RN. Concepts and practices in the evaluation of laboratory methods III: Statistics. Am J Med Technol 1978;44:552-71.

4 Stookey LL. Ferrozine: A new spectrophotometric reagent for iron. Anal Chem 1970;42:779-81.

5 Analytical methods for flame spectroscopy. Varian Publication No 85-100009-00, Springvale, Australia: Varian Techtron, 1979 .

6 Vogel AI. A textbook of quantitative inorganic analysis. 3rd edn. London: Longman, 1962:418. 